\title{
Radiation Tolerant Electronics and Digital Processing for the Phase-I Trigger Readout Upgrade of the ATLAS Liquid Argon Calorimeters
}

\author{
A. Milic, CERN, on behalf of the ATLAS Liquid Argon Calorimeter Group,
}

\begin{abstract}
The high luminosities of $\mathcal{L}>10^{34} \mathrm{~cm}^{-2} \mathrm{~s}^{-1}$ at the Large Hadron Collider (LHC) at CERN produce an intense radiation environment that the detectors and their electronics must withstand. The ATLAS detector is a multi-purpose apparatus constructed to explore the new particle physics regime opened by the LHC. Of the many decay particles observed by the ATLAS detector, the energy of the created electrons and photons is measured by a sampling calorimeter technique that uses Liquid Argon (LAr) as its active medium. The front end (FE) electronic readout of the ATLAS LAr calorimeter located on the detector itself consists of a combined analog and digital processing system. In order to exploit the higher luminosity while keeping the same trigger bandwidth of $100 \mathrm{kHz}$, higher transverse granularity, higher resolution and longitudinal shower shape information will be provided from the LAr calorimeter to the Level-1 trigger processors. New trigger readout electronics have been designed for this purpose, which will withstand the radiation dose levels expected for an integrated luminosity of $3000 \mathrm{fb}^{-1}$ during the high luminosity LHC (HL-LHC), which is well above the original LHC design qualifications.
\end{abstract}

\section{INTRODUCTION}

$\mathbf{T}$ HE ATLAS detector [1] operated very successfully during Run 1 (2009-2013) and collected a large number of events during proton-proton and heavy ion collisions, leading to important discoveries [2]. The calorimeters, in particular the LAr calorimeter, play an essential role in the energy measurement of all particles and also in the calorimeter trigger. One particular challenge when looking towards the future operation of the ATLAS detector and the LAr calorimeters is the limitation in the online event selection (Level-1 trigger) due to the existing trigger electronics with its constraints in granularity, bandwidth and latency. Without modifications, the peak instantaneous luminosity of $3 \times 10^{34} \mathrm{~cm}^{-2} \mathrm{~s}^{-1}$ expected during Run 3 (2018-2022), would force substantial increases in trigger thresholds because the detector will be operated in an environment with a mean number of pileup events of $\langle\mu\rangle=55$ per bunch crossing, as opposed to $\langle\mu\rangle \approx 20$ during Run 1. ATLAS is constrained by readout dead time to a $100 \mathrm{kHz}$ event rate at the Level-1 trigger. In order to handle the increased background rates to lepton triggers, the upgrade of the LAr calorimeter trigger readout aims at keeping the capability of triggering on low transverse energy $\left(E_{T}\right)$ electromagnetic (EM) objects while improving rejection of jets by using shower shape information. In the first section of this paper, the ATLAS calorimetry system and the current LAr calorimeter readout and trigger are described, followed

Manuscript received March 26, 2015. by the expected performance, design and radiation tests of the main components of the upgraded system. In the last section, first results measured with a demonstrator with the upgraded electronics installed on the detector are presented.

\section{ATLAS CALORIMETRY}

The ATLAS calorimetry system consists of several subdetectors, all fully symmetrical in $\phi$. They are designed to measure energy deposits of both charged and neutral particles within $|\eta|<4.9$. The structure of the ATLAS calorimeters is shown in Fig. 1.

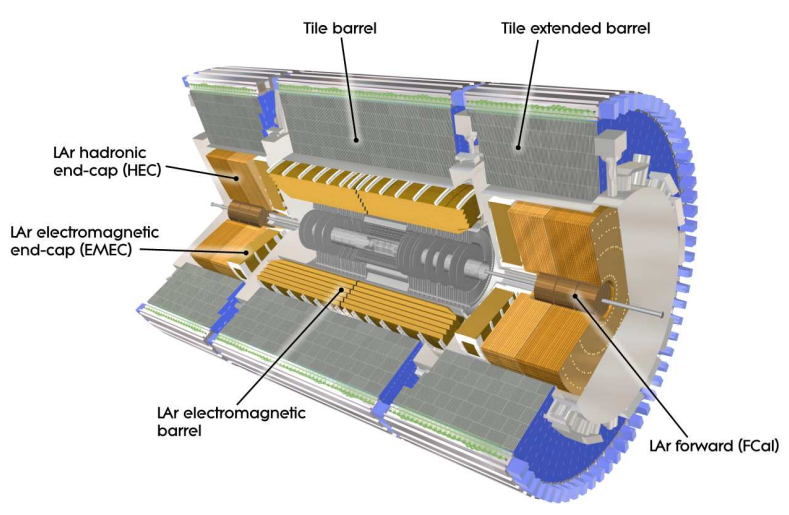

Fig. 1: The ATLAS calorimetry system with its subdetectors.

All ATLAS calorimeters are sampling calorimeters. Thus, absorber layers which initialize particle showers are alternated with layers of active material to perform energy measurements. An incident particle produces showers in stages, losing energy until the shower is completely absorbed. By summing up all measured energy within the calorimeter, the initial energy of the particle is reconstructed. In the EM calorimeter, the forward calorimeter (FCal) and the endcap of the hadronic calorimeter (HEC), LAr is used as active medium. In the barrel hadronic region scintillating tiles are used. LAr does not suffer from radiation damage as opposed to the tiles and is therefore preferable in the region close to the interaction point and the forward region. Since only a fraction of the shower energy absorbed by the active material can be measured, the calibration of the calorimetry system is crucially important.

\section{A. The LAr calorimeter in ATLAS}

The LAr barrel and endcap EM calorimeter is covering a pseudorapidity interval of $|\eta|<3.2$, the hadronic LAr 
calorimeter is ranging from $1.5<\eta<3.2$, and the FCal covers $3.1<\eta<4.9$. The central cryostat contains the EM barrel (EMB) calorimeter and the superconducting solenoid. Each endcap cryostat houses an EM Endcap (EMEC), two hadronic wheels and one FCal. The barrel of the LAr calorimeter is longitudinally seperated into layers: the presampler, front, middle and back layer. The cells in the respective layers have different granularity, as can be seen in the example of the EMB in Fig. 2. The accordion geometry is, among other factors, motivated by the aim to have full $\phi$ coverage with no cracks in between the module edges.

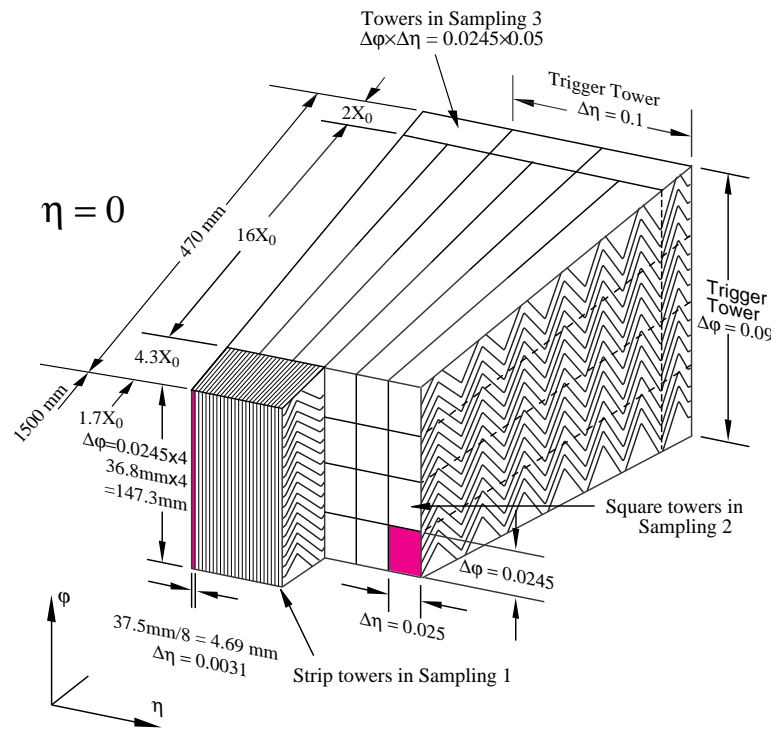

Fig. 2: Accordion structure and segmentation of the LAr calorimeter in the EM barrel.

In the EM barrel and the EMEC the absorbing material is lead, in the HEC copper is used. For the FCal, tungsten or copper rods, which form the cathode of the LAr cell, are set into an absorber matrix (copper for the EM module and tungsten for the two hadronic modules). Due to the high particle flux and positive ion build-up in the forward region the use of extremely narrow LAr gaps is required in order to avoid space charge building up and hence distortions of the drift field.

\section{B. Current electronics and Level-1 trigger}

All subdetectors deliver on their electrodes a triangularshaped current signal with a rise time of a few nanoseconds which falls to zero at the end of the drift time of ionization electrons in LAr, typically several hundreds of nanoseconds in the EM barrel. The current signal, which is typically about $2.8 \mu \mathrm{A} / \mathrm{GeV}$, is produced inside the detector.

The preamplifier is designed to cover the dynamic range in excess of 17 bits. This range is split by having a shaping amplifier with 3 linear output ranges with relative gains of approximately 1,10 and 100 . In that way the necessary dynamic range can be covered with a 12-bit system. For a typical cell capacitance of $1.5 \mathrm{nF}$ in the EM middle layer, the electronics noise is about $140 \mathrm{nA}$ which corresponds to about $50 \mathrm{MeV}$.
The coherent noise upper limit is $3 \mathrm{MeV} /$ readout channel. The critical points to be watched in order to achieve such low coherent noise is the grounding, the electrical power cabling and the extraction of dissipated heat. Switched capacitor arrays are used as an analog pipeline, covering a maximal latency time of $2.5 \mu \mathrm{s}$, followed by a multiplexed 12-bit ADC. The last stage of the calorimeter readout is the digital filtering, which extracts the best possible information from five samples which are digitized around the peak of the shaped signal. The corresponding boards are located besides the detector cavern. A digital serial optical link operating at $1.6 \mathrm{Gbps}$ connects them to the FE crates and transmits typically four or five samples per channel of Level-1 accepted events.

Signals for the Level-1 trigger are formed by analog sums and correspond to trigger units of $\Delta \eta \times \Delta \phi=0.1 \times 0.1$ (Trigger Towers). The summing is performed in three steps: on the shaper chips, on the Layer Sum Boards (LSB) of the FE Boards (FEB) and on the Tower Builder Boards (TBB). The resulting analog sums are transferred to the trigger receivers, which can equalize gain differences among channels, and then passed on to the digitizer system operating at $40 \mathrm{MHz}$.

\section{The ATLAS LAR CAlorimeter PhAse-I UPGRADE}

The existing calorimeter trigger information is based on the sum of the energy deposition across the longitudinal layers of the calorimeter in areas as large as $\Delta \eta \times \Delta \phi=0.1 \times 0.1$, the aforementioned Trigger Towers. The new finer granularity scheme is based on Super Cells, which provide information for each layer of the calorimeter and finer segmentation in the front and middle layer of the EMB and EMEC, as can be seen in Fig. 3.

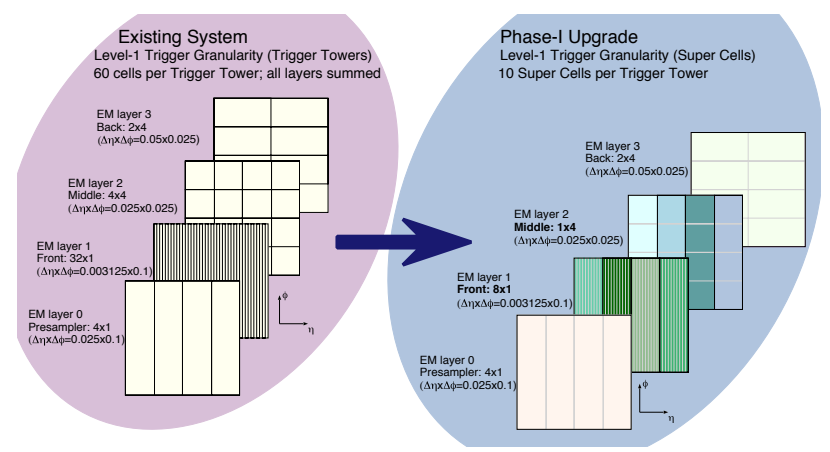

Fig. 3: Trigger Tower segmentation: In the presampler and back layer there is one Super Cell each, in the front and middle layer there are 4 Super Cells each.

The digitization precision of the Super Cell signals is improved by at least a factor of 4 to 10 compared to the existing Level-1 system. The online calculation of the transverse energy deposited in the Super Cells will provide results close to the energy resolution obtained from the current offline reconstruction.

\section{A. Expected performance of the upgraded system}

The finer granularity of the Super Cells will improve the trigger energy resolution and efficiency for selecting electrons, 
photons, $\tau$ leptons, jets and missing transverse momentum, while enhancing the discrimination against hadronic jets and pileup. Rejecting jet backgrounds to EM triggers will be possible in a more sophisticated way through the use of the following shower shape variables at the Level-1 stage:

- $R_{\eta}$ is defined as the transverse energy measured in the $\eta \times \phi=3 \times 2$ group centered around the Super Cell with the highest energy in the middle layer divided by the transverse energy measured in a $\eta \times \phi=7 \times 2$ group.

- $f_{3}$ is the ratio of the transverse energy measured in the back EM layer to that deposited in all three layers for an EM cluster.

- $w_{\eta, 2}$ is the spread of the shower in the middle EM layer in a $3 \times 2$ Super Cell region, defined as an energy weighted root mean square.

In addition to these variables, hadronic isolation $\left(E_{\text {had }}^{\text {core }} \leq 1\right.$ $\mathrm{GeV}$ ) can be required for each electron candidate, where $E_{\text {had }}^{\text {core }}$ is defined as the transverse energy deposited in a $\Delta \eta \times \Delta \phi=0.2 \times 0.2$ region of the hadronic calorimeter behind the EM shower. A multi-dimensional optimization is performed to minimize the trigger rate while keeping a high signal efficiency. In Fig. 4 the distributions of $R_{\eta}$ and $f_{3}$ with the upgraded Level-1 trigger are shown for a pileup of $\langle\mu\rangle=80$. They allow to distinguish between electrons and jets with $p_{T}>20 \mathrm{GeV}$ and at the same time keep a high trigger efficiency.
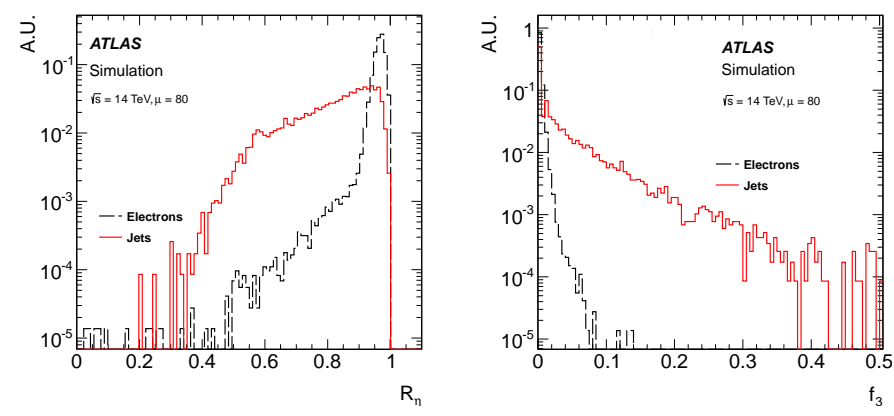

Fig. 4: Distributions of $R_{\eta}$ and $f_{3}$ for electrons and jets, both normalized to unit area.

Fig. 5 provides a comparison of the Level-1 trigger thresholds and rates anticipated in Run 2 to that of the upgraded Level-1 trigger for a $95 \%$ electron efficiency in both cases. For a given bandwidth of e.g., $20 \mathrm{kHz}$, the Level-1 threshold, which would need to be $28.5 \mathrm{GeV}$ assuming Run 2 conditions, could be lowered by $7 \mathrm{GeV}$ to $21.5 \mathrm{GeV}$ with the Phase-I upgrade.

\section{B. Design of the new electronics}

The upgrade of the trigger readout begins with rerouting the signals from the linear mixer in the shaper chips of the FE electronics to the new LSBs, which produce finer granularity Super Cell signals for the front and middle layers of the calorimeter. The new baseplane will transfer those Super Cell signals to the LAr Trigger Digitizer Boards (LTDB).

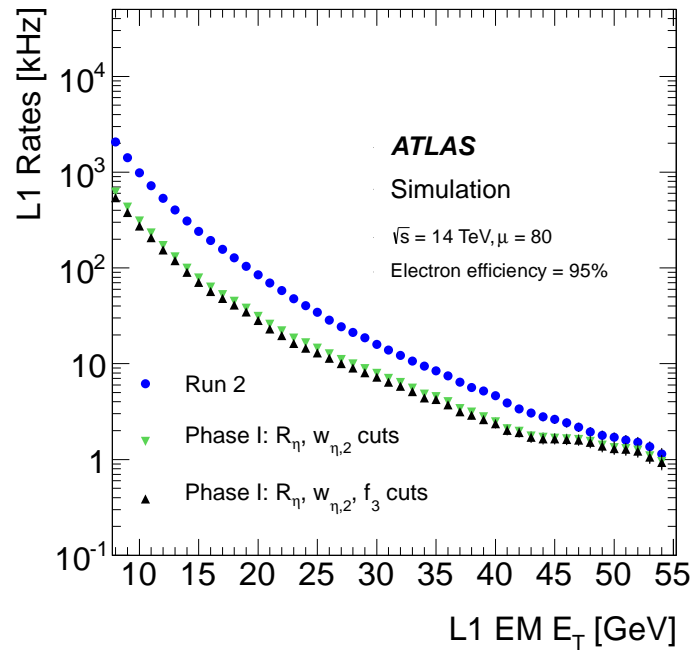

Fig. 5: Level-1 trigger rates for two sets of cuts, as measured from a sample of simulated minimum bias events with a pileup of $\langle\mu\rangle=80$.

The LTDB digitizes the Super Cell signals and sends the samples to the new back end (BE) electronics located in the cavern besides the detector. Then they are transmitted to the trigger processors. Each LTDB will process up to 320 Super Cell signals. The signals will be digitized by 12-bit ADCs on the LTDB. Data of 8 ADC channels will be serialized and transmitted at $5.12 \mathrm{Gbps}$ to the BE electronics. Each LTDB will typically have 40 fibers and deliver 25 Tbps digital information to the boards in the BE system. This system is made up of $31 \mathrm{LAr}$ Digital Processing Blades (LDPB) housed in three ATCA shelves. Each LDPB consists of one carrier board equipped with four Advanced Mezzanine Cards equipped with powerful FPGAs which will process the data of up to 320 Super Cells each.

For the Phase-I upgrade the old legacy Level-1 trigger system will be kept operational during the start-up. This is done by the LTDB recreating the analog sums of the Trigger Towers and feeding them back to the TBB.

In Fig. 6 the layout of the current and upgraded FE and BE electronics is shown.

The thresholds of the Level-1 trigger system are proportional to the transverse energy, whereas the calorimeter signals are proportional to the energy. Currently the conversion factor of $\sin \theta$ is applied in the TBB for the EM calorimeters (through the $\eta$-dependent gains) and in the receiver for the HEC and FCal. It has not been decided yet how the $\eta$-dependent gains will be applied in the new electronics. They may be included in the analog section of the LTDB or digitally in the BE electronics. A detailed description of all components of the upgrade electronics can be found in [3].

The energy deposited by particle showers in the calorimeter cells has to be reconstructed and assigned to the correct bunch crossing. This is not always straightforward, as e.g. for saturated pulses dedicated algorithms have to be implemented to assign a certain energy to the precise bunch crossing. The energy reconstruction algorithm is based on a linear 


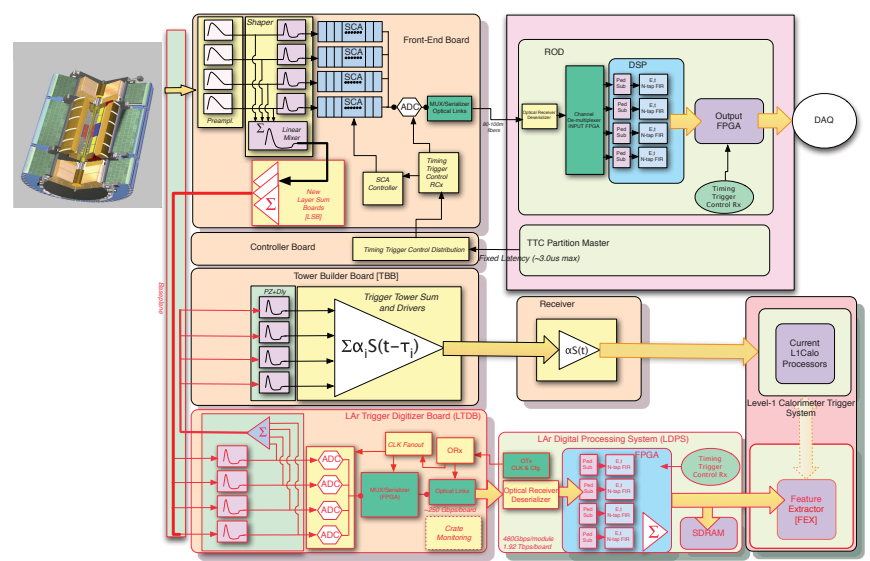

Fig. 6: Architecture of the Phase-I upgrade LAr trigger readout. The new components are indicated by the red outlines and arrows.

combination of the signal samples. The coefficients minimize electronics and pileup noise by having precise knowledge of the pulse shape. As luminosity increases, pileup becomes the dominant contribution to the total noise in most calorimeter regions. Fig. 7 shows the total noise expected for different calorimeter sections.

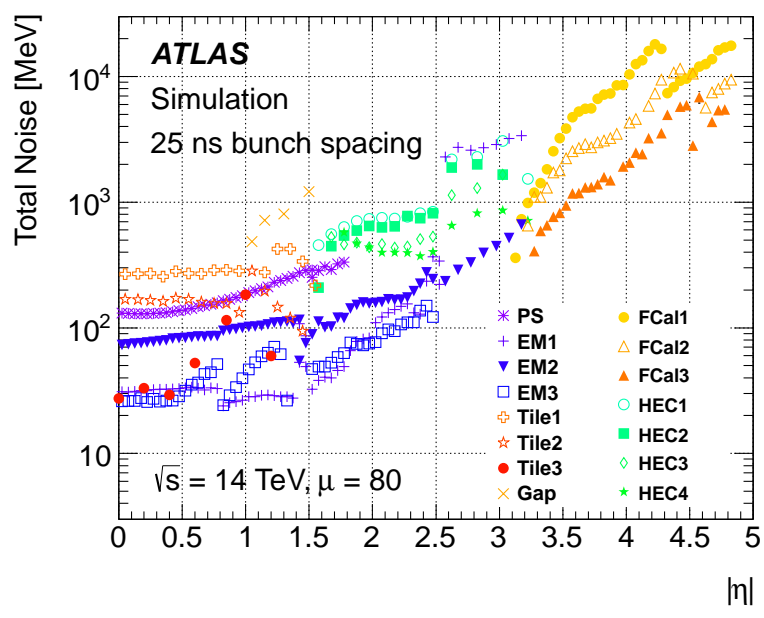

Fig. 7: Simulated noise of the transverse energy per cell in all layers of the LAr and Tile calorimeter as a function of $|\eta|$. The pileup is $\langle\mu\rangle=80$ corresponding to the nominal Run 3 pileup conditions.

\section{RADIATION HARDNESS TESTS}

Radiation in the ATLAS detector is dominated by secondary particles produced by interactions of the primary particles with the detector elements. As a result, at the electronics location, the energies are rather low (less than a few $\mathrm{GeV}$ ), the fluxes are high, and the radiation field is roughly homogeneous. The dominant contributions to the flux consists of photons and neutrons, but also charged hadrons such as protons and pions. A high level of reliability of the electronics must be maintained during the estimated seventeen years of operation of the experiment.
ADCs, op-amps, voltage references and low-dropout (LDO) regulators with low supply voltages intended to be used for the upgrade were tested to see if they might hold up under high levels of proton and gamma irradiation.

The ADC is mounted on the LTDB. It must use less than $145 \mathrm{~mW}$ per channel, must be radiation tolerant up to $\approx 150$ kRad and have low Single Event Effect (SEE) sensitivity. More detailed design specifications for the ADC can be found in [4].

For the op-amps, voltage references and LDO regulators a voltage change of less than $10 \%$ after an exposure of $100 \mathrm{kRad}$ was considered as sufficient.

Numerous devices were tested by irradiation with proton and gamma radiation. Here, just a few results of devices which passed the requirements, are presented.

For the proton irradiation a $230 \mathrm{MeV}$ proton beam was used. During the ADC tests, the chips were powered and a clock signal was applied, while the current drawn by the chip was monitored. A sine-wave signal was provided as input to the ADC. Over the course of the irradiation well beyond the requirement, the Effective Number Of Bits (ENOB) remained stable, as can be seen in Fig. 8 .

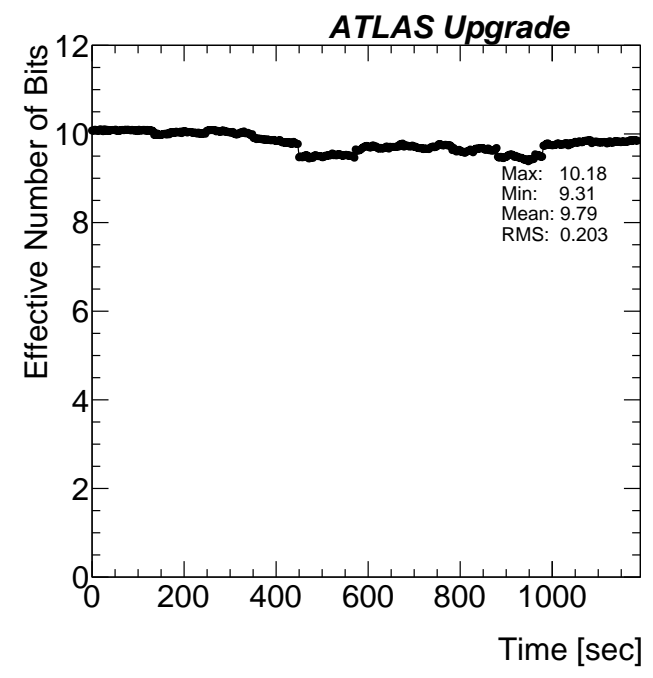

Fig. 8: ENOB measured during proton irradiation of the ADC considered for being used for the upgrade electronics. At the end of the exposure the Total Integrated Dose (TID) was 2 MRad.

The LDO regulator for which the voltage change during irradiation is shown in Fig. 9 is a good example of a voltage regulator not affected by protons. The change in voltage was $2.25 \%$ from the nominal $2.43 \mathrm{~V}$ to $2.45 \mathrm{~V}$ after an exposure of $2.65 \times 10^{11}$ protons $/ \mathrm{cm}^{2}$.

As mentioned before, several LDO regulators, op-amps and voltage references were also exposed to gamma irradiation from a ${ }^{60} \mathrm{Co}$ source. The first irradiation run took 20 hours, resulting in an exposure of $100 \mathrm{kRad}$, followed by an annealing period of 75 hours. The second irradiation lasted 40 hours for an additional $200 \mathrm{kRad}$, followed by another annealing period of 50 hours. Fig. 10 shows one of the regulators tested which remained operational up to an exposure of $168 \mathrm{kRad}$. 


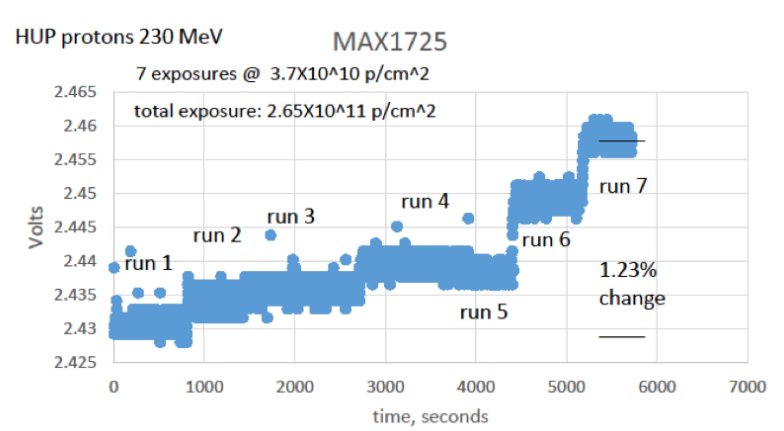

Fig. 9: Voltage change of the MAX1725 LDO regulator after an irradiation of $2.65 \times 10^{11}$ protons $/ \mathrm{cm}^{2}$.

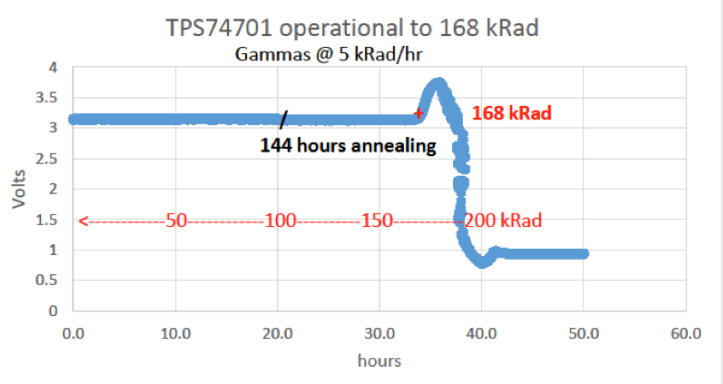

Fig. 10: Voltage change of the TPS74701 regulator for a gamma irradiation of up to $300 \mathrm{kRad}$.

\section{INSTALLATION AND TESTS OF THE DEMONSTRATOR SYSTEM IN ATLAS}

In order to test the new electronics components, a test setup in the lab was established with a half FE crate equipped with boards and a readout system equivalent to the one operating in ATLAS. The calorimeter cells were simulated by a load which was plugged at the back of the baseplane. The calibration system was used for injecting signals into the FEBs. After successful tests of the demonstrator system in our lab and validation of the new baseplane and the LTDB prototypes, the demonstrator was installed in ATLAS in one of the crates of the EMB (Fig. 11) [5]. While the demonstrator is meant to test the functionality of the future Phase-I upgrade, some nonradiation hard components are used for these pre-prototype boards (FPGA, commercial optical links).

In order to verify the proper installation of the demonstrator, calibration data were taken both via the standard readout and with the LTDB prototypes. Tests verifying that the total and coherent noise levels and the amplitude shapes of the FEBs are the same as in the adjacent crates were performed. In Fig. 12 the total noise on every readout channel of the FEBs of the demonstrator crate is compared to the total noise of its neighboring crate. As can be seen, noise levels are the same. The FEBs read out signals from different layers of the calorimeter. The noise levels of the boards vary because different capacitances and gains are applied to their respective cells. Since the two crates read out adjacent regions in the calorimeter, the noise is expected to be similar.

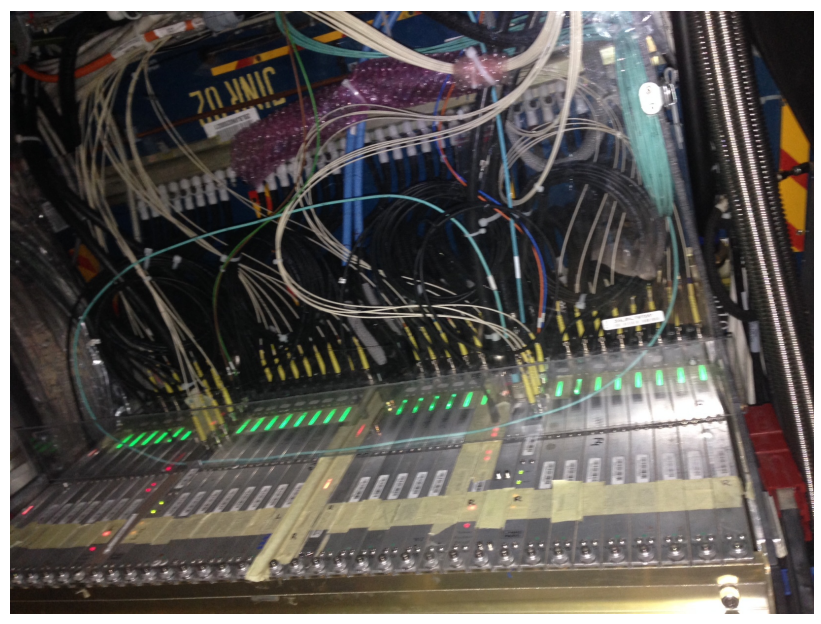

Fig. 11: Full crate installed on the ATLAS detector equipped with all FEBs and the LTDB.

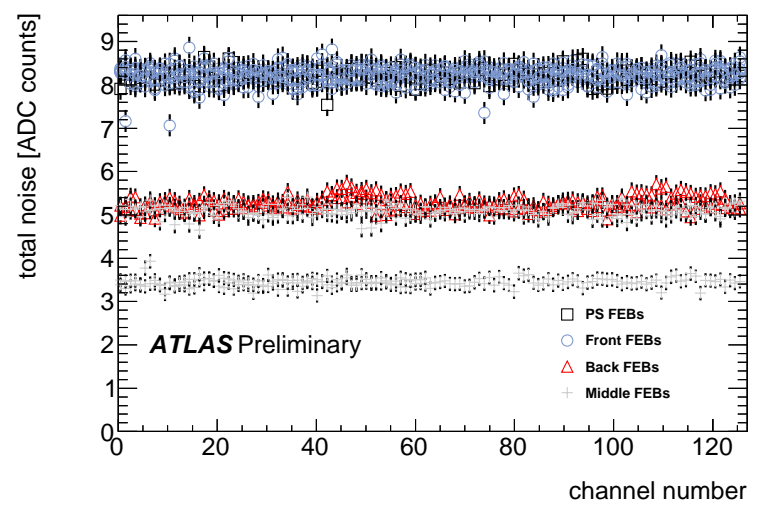

(a)

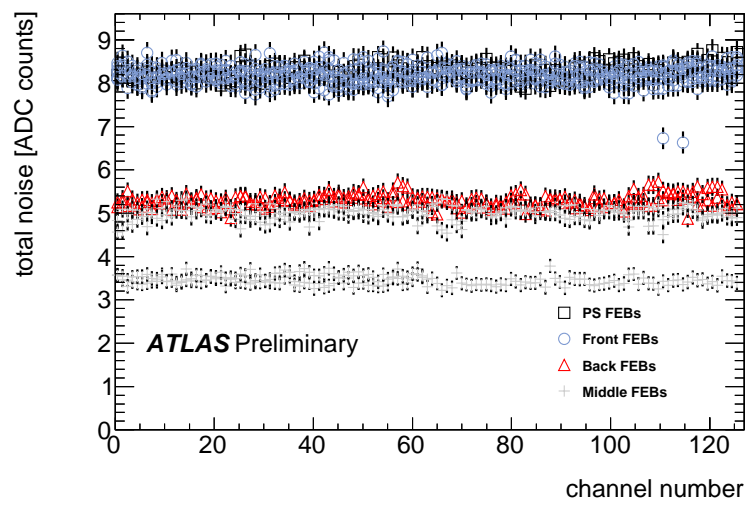

(b)

Fig. 12: Comparison of the total noise of the demonstrator crate (a) and the total noise of its adjacent crate (b).

For the LTDB installed in the demonstrator crate, the pedestal value and total noise of the 12-bit ADC of the 320 channels were measured (Fig. 13). One ADC count corresponds to roughly $125 \mathrm{MeV}$.

In our test setup on the surface, we could also measure a pulse sent to the LTDB by the calibration system (Fig. 14). 


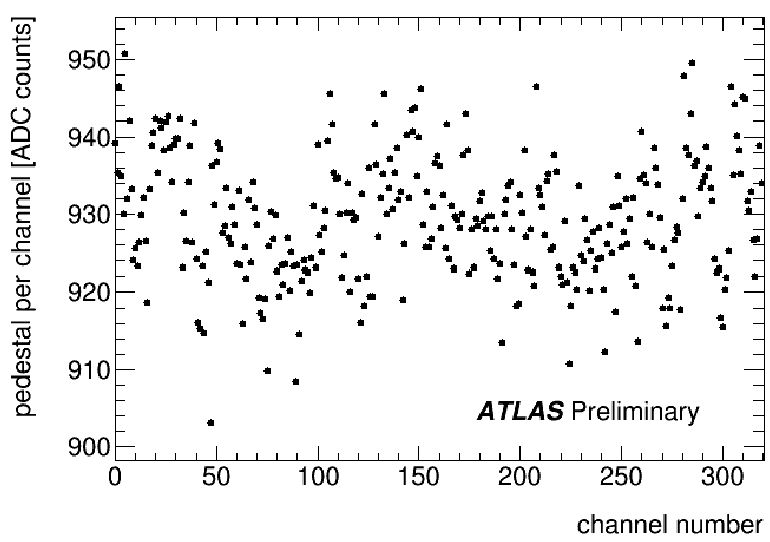

(a)

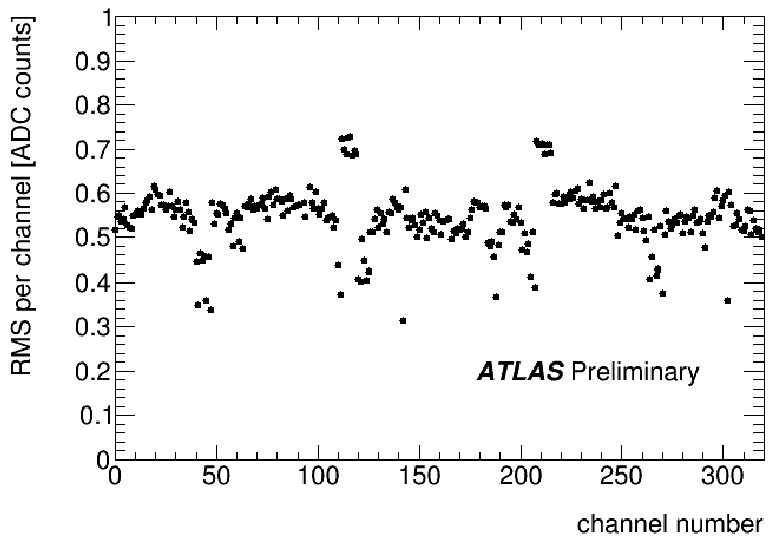

(b)

Fig. 13: Pedestal (a) and total noise (b) of the 12-bit ADC measured for all 320 channels on the LTDB.

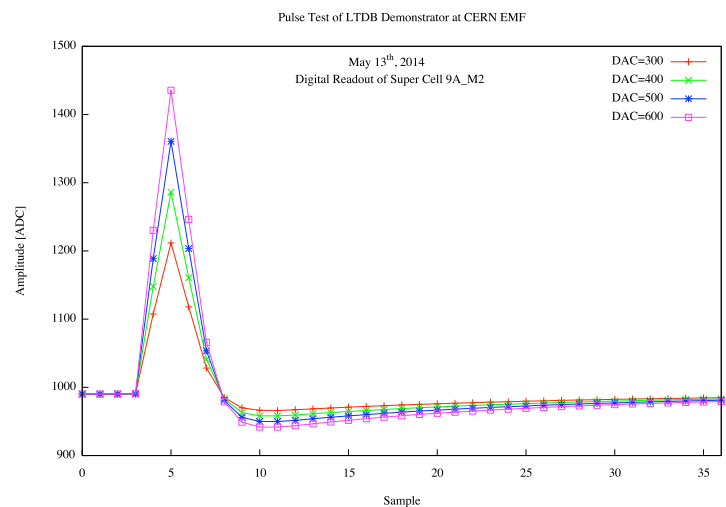

Fig. 14: One channel of the FEBs was pulsed with four different amplitude values. The response of one of the LTDB channels which corresponds to one Super Cell is shown in this plot. The number of samples is adjusted to cover the pulse.

A further consideration was the fraction of the noise that was coherent among all channels of the LTDB. In the following this is refered to as coherent noise fraction (CNF). It has to be kept low because it can introduce fake energy in large areas of the detector. According to the ATLAS requirement, the coherent noise has to be kept under $5 \%$ in the LAr calorimeter.
In Fig. 15 the CNF is shown per FEB for all feedthroughs (FT). The CNF for a large area of the EMB has been computed. FT 9 and 10 belong to the crate where the demonstrator is installed. FT 7, 8, 11 and 12 belong to adjacent FE crates. The board in the first slot of the FE crate reads out the presampler, the boards in the following seven slots read out the front layer, the next two boards the back layer and the last four boards the middle layer of the calorimeter. The last entry is the CNF of the whole half-crate. All values are below the required $5 \%$ level.

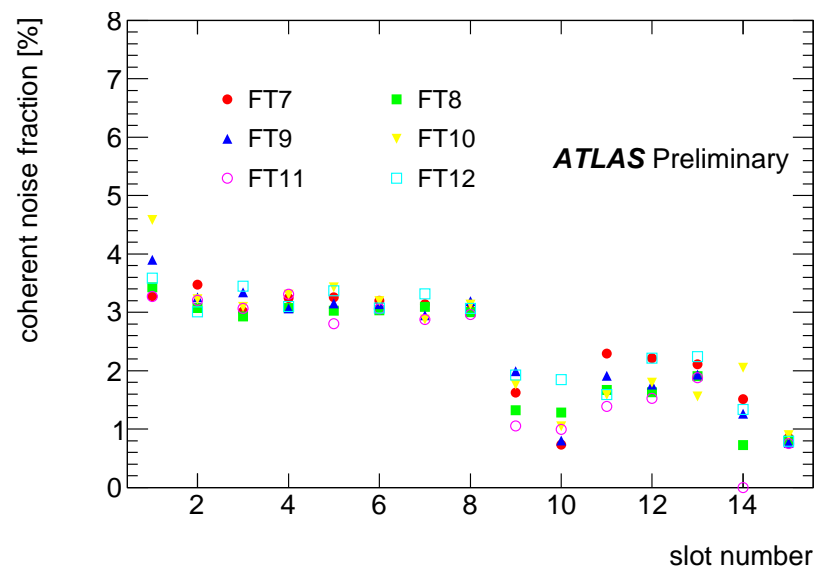

Fig. 15: Coherent noise fraction (CNF) for the FEBs in FT 7-12 on the EMB.

\section{CONCLUSIONS}

With the upgraded LAr calorimeter trigger readout which will be operating in ATLAS after the LHC Phase-I upgrade, a finer segmentation of the calorimeter will be made available at the trigger level. This will help to reduce the QCD jet background in electron, photon and $\tau$ lepton triggers and improve jet and missing $E_{T}$ trigger performance. It will enable ATLAS to retain low trigger thresholds when it is operated at a luminosity of $3 \times 10^{34} \mathrm{~cm}^{-2} \mathrm{~s}^{-1}$ and beyond. During Run 2, it will be possible to measure the performance of the new readout system with the demonstrator installed on the detector and gain experience for the Phase-I upgrade, when all FE crates will be equipped with the new electronics. The upgraded trigger readout is planned to be used as the low-latency Level-0 trigger after the Phase-II upgrade (2022-2024), during which a fully digital LAr FE readout will be installed [6].

\section{REFERENCES}

[1] ATLAS Collaboration, The ATLAS Experiment at the CERN Large Hadron Collider, 2008 JINST 3 S08003

[2] ATLAS Collaboration, Observation of a new particle in the search for the Standard Model Higgs boson with the ATLAS detector at the LHC, Phys.Rev. D88 (2013) 032004

[3] ATLAS Collaboration, ATLAS Liquid Argon Calorimeter Phase-I Upgrade Technical Design Report, CERN-LHCC-2013-017 (2013), http://cds.cern.ch/record/1602230

[4] J. Kuppambatti et. al., A Radiation-Hard Dual-Channel 12-bit $40 \mathrm{MS} / \mathrm{s}$ ADC Prototype for the ATLAS Liquid Argon Calorimeter Readout Electronics Upgrade at the CERN LHC, 2013 JINST 8 P09008

[5] A. Milic, Demonstrator for the ATLAS LAr calorimeter Phase-I Trigger Readout Upgrade, http://cds.cern.ch/record/1994994

[6] O. Novgorodova, The ATLAS liquid argon calorimeter: upgrade plans for the HL-LHC, J. Phys.: Conf. Ser., Vol. 587 (2015) 